\title{
Chromatin-remodeling enzymes in control of Schwann cell development, maintenance and plasticity Claire Jacob
}

\begin{abstract}
Gene regulation is essential for cellular differentiation and plasticity. Schwann cells (SCs), the myelinating glia of the peripheral nervous system (PNS), develop from neural crest cells to mature myelinating SCs and can at early developmental stage differentiate into various cell types. After a PNS lesion, SCs can also convert into repair cells that guide and stimulate axonal regrowth, and remyelinate regenerated axons. What controls their development and versatile nature? Several recent studies highlight the key roles of chromatin modifiers in these processes, allowing SCs to regulate their gene expression profile and thereby acquire or change their identity and quickly react to their environment.
\end{abstract}

\section{Address}

Department of Biology, University of Fribourg, Chemin du Musée 10, 1700 Fribourg, Switzerland

Corresponding author: Jacob, Claire (claire.jacob@unifr.ch) because it contains growth inhibitory proteins [5]. However, SCs react quickly to an axonal lesion by dedifferentiating, digesting their own myelin - a process called myelinophagy [6] — and converting into repair cells $[7,8]$ that foster axonal regrowth and guide axons back to their former target $[9,10]$. SCs then remyelinate regenerated axons (Figure 2). This remarkable SC plasticity allows the PNS to functionally regenerate after lesion.

This review is focused on the mechanisms of SC development, maintenance and plasticity after lesion controlled by chromatin-remodeling enzymes. Chromatin remodeling regulates the accessibility of genes for the transcriptional machinery, and thereby gene activation and repression. Changes of chromatin architecture are controlled by ATP-dependent nucleosome remodeling and by covalent modifications, either on DNA by methylation or on histones by various post-translational modifications including acetylation, methylation, phosphorylation, ubiquitination, SUMOylation, ADP-ribosylation. Although our knowledge on these mechanisms is still very sparse, recent findings on the functions of chromatinremodeling enzymes have significantly contributed to a better understanding of their critical functions in SCs.

\section{Chromatin-remodeling factors controlling SC development, maintenance and plasticity after lesion}

Nucleosome-remodeling complexes, DNA methylation/ demethylation, histone acetylation/deacetylation and methylation/demethylation enzymes have been shown to hold key functions in SCs (Figures 1 and 2).

\section{Nucleosome-remodeling complexes}

Nucleosome-remodeling complexes use ATP to destabilize the interaction between DNA and histones. This local chromatin destabilization results in nucleosome sliding and repositioning that can also lead to adjacent nucleosome ejection or insertion. These changes of chromatin structure modify DNA accessibility, and can lead to either transcriptional activation or repression. Nucleosome-remodeling complexes are classified into different families, based on their composition and activity: SWI/ SNF, ISWI, INO80/SWR1 and NuRD complexes [11]. Among those, the SWI/SNF and the NuRD complexes are known to regulate aspects of SC development and plasticity after lesion (Figures 1 and 2). Two subtypes BAF and PBAF of SWI/SNF complexes are known; they owe their enzymatic activity to their ATPase subunit BRM or BrG1 [12]. The NuRD complex comprises 


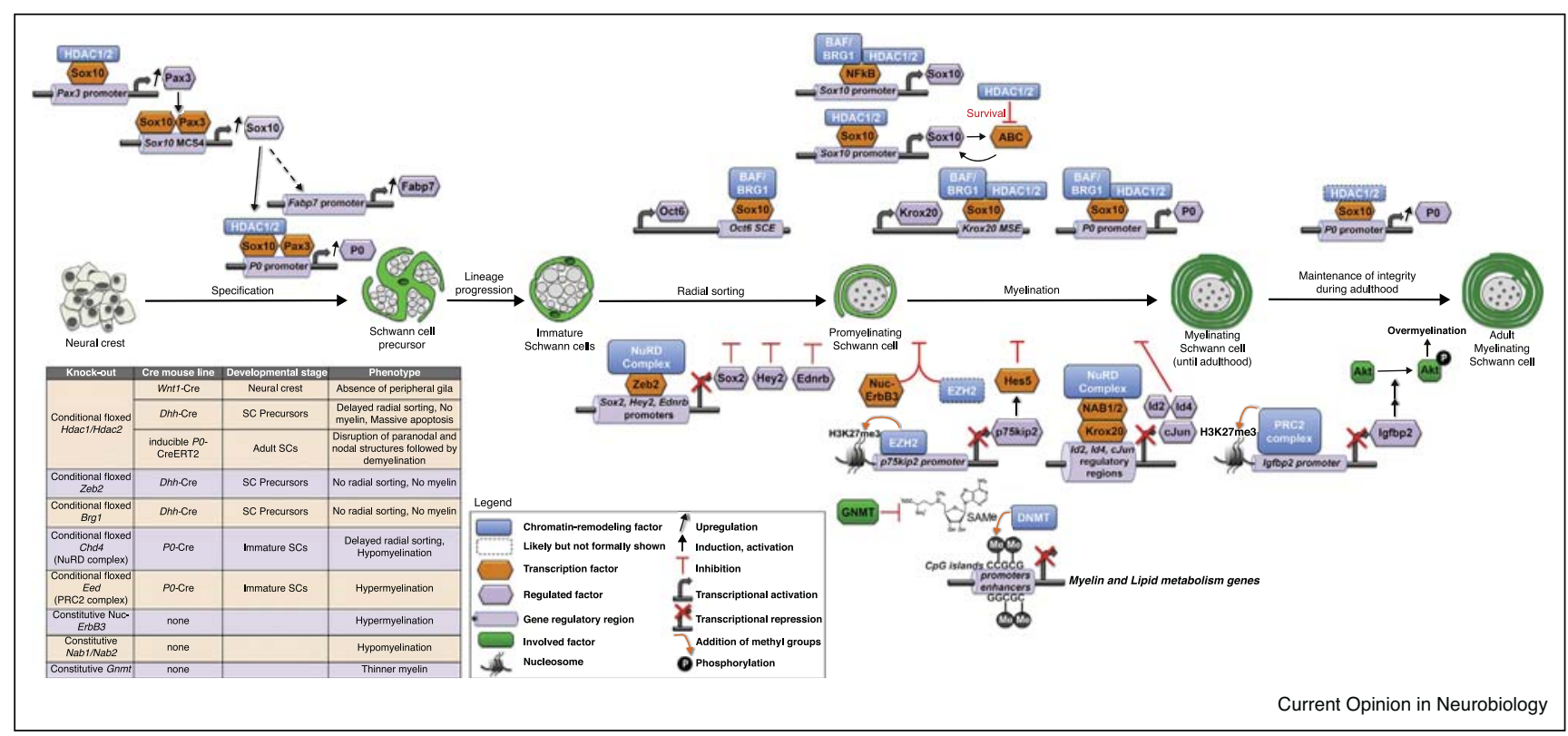

Chromatin-remodeling enzymes and associated mechanisms regulating SC development and maintenance. Activating mechanisms are depicted above and inactivating mechanisms underneath SC stages. The table lists mouse mutants and their phenotypes used to identify these mechanisms.

Figure 2

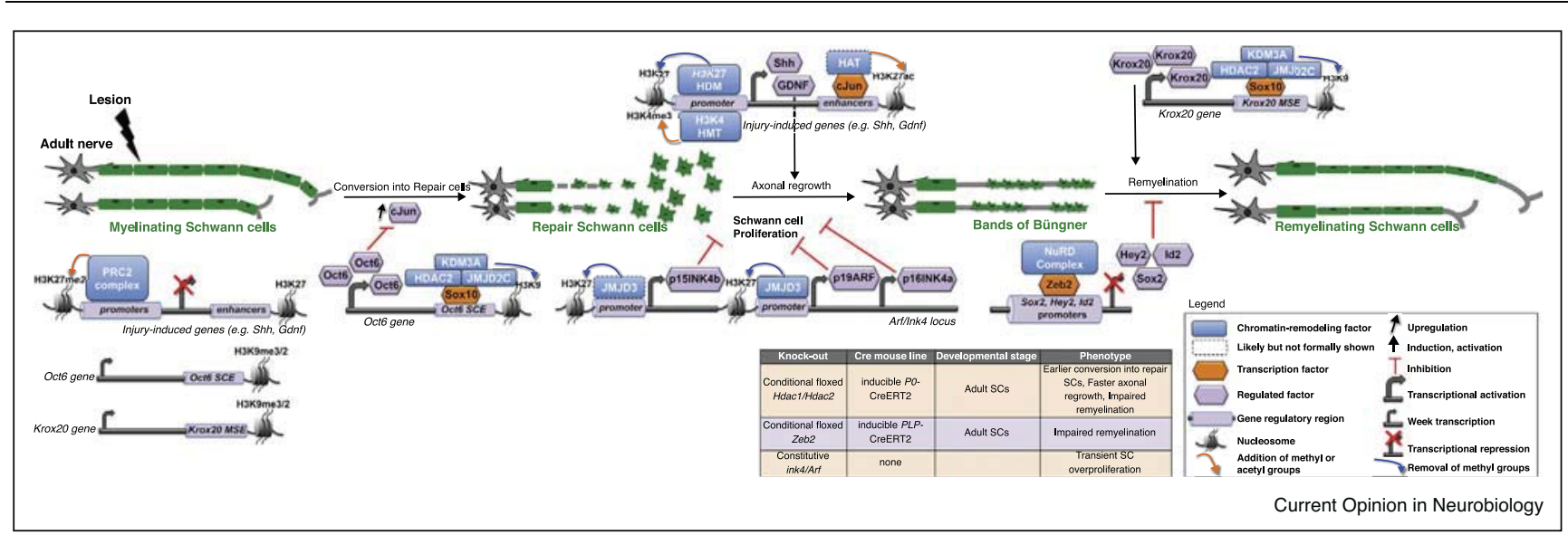

Chromatin-remodeling mechanisms regulating SC plasticity after lesion. Activating mechanisms are depicted above and inactivating mechanisms underneath regeneration stages. The table lists mouse mutants and their phenotypes used to identify these mechanisms.

CHD3 or CHD4 as ATPase subunit, and has in addition deacetylase activity by the recruitment of HDAC1 and HDAC2 [13].

\section{DNA methyltransferases (DNMTs)}

DNMTs transfer a methyl group from a methyl donor, generally $\mathrm{S}$-adenosylmethionine (SAMe) to the carbon 5 of cytosines located within $\mathrm{CpG}$ dinucleotides [14]. Most CpGs in mammalian genomes are maintained methylated across generations by DNMT1. By contrast, $\mathrm{CpG}$ islands in promoters and enhancers often remain unmethylated through active DNMTs exclusion. This usually requires recruitment of a transcription factor and other partners such as histone H3 lysine 4 (H3K4) methyltransferases (HMTs), a TET dioxygenase and a thymidine DNA glycosylase that erase/prevent methylation. Promoter silencing can however occur through de novo methylation at $\mathrm{CpG}$ islands by DNMT3A or DNMT3B, 
and is usually preceded by repressive H3K9 methylation marks. Interestingly, in the case of very low availability of methyl donor, DNMT3A/B cannot methylate CpGs, but in contrast have been reported to actively participate in DNA demethylation [15].

\section{Histone acetylation and deacetylation enzymes}

Histone acetyltransferases (HATs) add acetyl groups to lysines of histone tails, which loosens the attraction between histones and DNA and results in chromatin decompaction that facilitates the access for the transcriptional machinery. By contrast, HDACs remove these acetyl groups, which leads to a more condensed chromatin that restricts DNA access [16]. HATs are thus commonly thought to act as transcriptional co-activators, whereas HDACs as transcriptional co-repressors. However, several studies have shown that HDACs can also participate in transcriptional activation [17-19]. HATs and HDACs cannot bind DNA directly and thus need a DNA-binding partner to modify histones. In addition, these enzymes can acetylate and deacetylate and thereby control the activity of non-histone targets including several transcription factors [20]; they are thus very powerful transcriptional regulators. Eighteen known mammalian HDACs are subdivided into four classes, based on their structure [21]. HDAC1 and HDAC2, two highly homologous class I HDACs that can usually compensate each other's functions, have been extensively studied in SCs.

\section{Histone methylation and demethylation enzymes}

HMTs catalyze the addition of methyl groups to target residues, whereas histone demethylases (HDMs) remove these methyl groups. Histones can be methylated on the three basic residues $\mathrm{K}$, arginine ( $\mathrm{R}$ ) and histidine (His), although His methylation is rare. Activating methylation marks are located on H3K4, H3K36, H3K79, H3R17 and H3R26, and repressive methylation marks on H3K9, H3K27, H4K20 and H3R8, whereas methylation of H3R2 and H4R3 leads either to transcriptional activation or repression, depending on the exact location of the methyl group. There are several families of HMTs and K HDMs, but the existence of R HDMs is not clear [22].

\section{Functions of chromatin-remodeling enzymes in SC development and maintenance of PNS integrity}

SC development from specification of the lineage to terminal differentiation into myelinating cells and maintenance of myelination require transcriptional activation of genes that induce lineage differentiation and acquisition and maintenance of cell stage identity. HDAC1 and HDAC2 (HDAC1/2) and the BAF complex hold major functions in these processes. For differentiation to proceed, inactivating inhibitory mechanisms of SC differentiation is also necessary. In addition, preventing overmyelination is required for stable myelination and optimal nerve conduction. The NuRD complex as well as DNA and histone methylation regulate these mechanisms. Current knowledge on this topic is discussed below and summarized in Figure 1.

\section{HDAC1/2 control SC lineage specification}

The transcription factor Sox10 is essential for SC lineage specification and the entire SC developmental process [23]. However, Sox10 is expressed in all neural crest cells. Thus, additional mechanisms direct Sox10-dependent specification into the SC lineage. Indeed, ablation of HDAC1/2 in neural crest cells by crossing Hdac1/2 floxed mice with mice expressing the Cre recombinase (Cre) under control of the Wnt1 promoter (Wnt1-Cre) prevents peripheral glia specification [24]. We show that HDAC1/2 and Sox 10 interact to activate the promoter of the early lineage marker Myelin protein zero (PO [25]) and of Pax3, another key transcription factor for SC specification [26,27]. In turn, Pax3 and Sox10 activate the Sox10 MCS4 enhancer (also called U3 [28,29]) to maintain high levels of Sox 10, which induces expression of $P O$ and Fatty acid binding protein 7 ( $F a b p 7)$, another early peripheral glia marker [30].

\section{HDAC1/2 regulate radial sorting, SC survival, induction} and maintenance of myelination

HDAC1/2 have also essential functions at later developmental stages and in the maintenance of integrity in adult nerves. Ablation of HDAC1/2 in SC precursors (by crossing Hdac1/2 floxed mice with mice expressing Cre under control of the Dhh promoter) leads to radial sorting delay and virtual absence of myelin, followed by massive SC apoptosis [31]. We showed that HDAC1/2 maintain SC survival by limiting active-beta-catenin $(\mathrm{ABC})$ levels and that they interact with Sox 10 to activate the Sox 10 and $P O$ promoters and the Krox20 MSE (myelinating SC element [32]), a critical enhancer for expression of the major transcription factor of SC myelination Krox20 [33]. In adult SCs, ablation of HDAC1/2 (using the tamoxifeninducible CreERT2 under control of the $P O$ promoter) leads to disruption of paranodal and nodal structures, followed by moderate demyelination due to $50 \%$ loss of P0 expression, presumably caused by impairment of Sox10-mediated activation of the $P O$ promoter [34]. Chen et al. [35] also showed that HDAC1/2 interact with the transcription factor NFkB to activate the Sox 10 promoter in SCs during developmental myelination. However in vivo NFkB inactivation leads to minor effects on myelination [36], therefore this mechanism may have a minor control over in vivo myelination.

\section{The BAF complex ATPase BrG1 is required for radial sorting and myelination}

Ablation of BrG1 in SC precursors (using floxed Brg1 and Dhh-Cre mouse lines) prevents radial sorting and myelination [37,38]. Limpert and colleagues (2013) show that BrG1 interacts with $\mathrm{NFkB}$ to activate the Sox10 promoter and suggest that this mechanism controls BrG1-dependent SC 
myelination [37]. However, given the minor effect of in vivo NFkB inactivation on myelination [36], BrG1 interaction with $\mathrm{NFkB}$ is likely to have minor functions on $\mathrm{SC}$ myelination. Furthermore, while Sox10 levels are moderately [37] or not [38] affected in the absence of BrG1 in SCs, Oct6, Krox 20 and P0 are virtually absent. Indeed, the BAF complex is recruited by Sox10 to the Oct6 SCE (SC enhancer [39]), the Krox20 MSE and the $P 0$ promoter to induce Oct6, Krox20 and P0 expression [38-40], and thereby controls the myelination process.

\section{The NuRD complex regulates radial sorting and myelination}

As mentioned earlier, Krox20 is a major transcription factor of SC myelination, which is absolutely required for the activation of myelin genes and the production of peripheral myelin [33]. Interestingly, Krox20 can also act as a transcriptional repressor. Indeed, Krox20 can interact with the transcriptional repressors $\mathrm{NAB} 1 / 2$, which recruit the NuRD complex to silence expression of inhibitors of myelination including Id2, Id4 and $c J u n$ at a late myelination stage [41-43]. Consistently, ablation of the NuRD complex ATPase CHD4 in immature SCs (using floxed Chd4 and PO-Cre mouse lines) leads to increased expression of inhibitors of myelination and to hypomyelination [43]. Ablation of CHD4 also leads to delayed radial sorting [43]. At this earlier developmental stage, the NuRD complex may allow radial sorting by silencing the transcription of Sox2, Hey2 and Ednrb $\left[44^{\bullet \bullet}, 45^{\bullet \bullet}\right.$, other inhibitors of SC differentiation, together with the transcription factor Zeb2. Indeed, Zeb2 has been shown to recruit the NuRD complex in SCs [44 ${ }^{\circ \bullet}$ ] and ablation of Zeb2 in SC precursors (using floxed Zeb2 and Dhh-Cre mouse lines) leads to upregulation of Sox2, Hey2 and Ednrb and prevents radial sorting and myelination $\left[44^{\bullet \bullet}, 45^{\bullet \bullet}\right]$. However, ablation of Zeb2 results in a more severe phenotype as compared to ablation of CHD4 [43], suggesting that Zeb2 acts also through CHD4-independent mechanisms. Alternatively, the different levels of phenotype severity between Zeb2 and Chd4 mutant mice could be due to the different Gre mouse lines used to generate these mutants.

\section{DNA demethylation adjusts myelin thickness}

DNA demethylation of selected genes appears necessary for appropriate developmental myelination. Indeed, DNA demethylation at promoters and enhancers of myelin genes $(M b p, \operatorname{Pmp} 22, \operatorname{Prx}, C n p)$, transcriptional regulators of myelination (Lgi4, Nab1, Nfatc1) and lipid metabolism genes (Scap, Srebf1, Hmgcr, Mvk, Pmvk, Dgat1, Lipe, Scd1, Abca2 and Elovl7) correlates with upregulation of these genes during myelination $\left[46^{\bullet \bullet}\right.$. Simultaneously, expression of DNMTs decreases and expression of Gadd45a (growth arrest and DNA damage 45a), Gadd45b, and Apobec1 (apolipoprotein B mRNA editing enzyme catalytic subunit 1), which promote DNA demethylation [47], increases [ $46^{\bullet \bullet}$ ]. The principal methyl donor SAMe is converted into S-adenosylhomocysteine and N-methylglycine by Glycine N-methyltransferase (GNMT, [48]). Ablation of GNMT impairs this conversion and thus increases the availability of SAMe, which results in a thin myelin phenotype in constitutive Gnmt mutant mice $\left[46^{\bullet \bullet}, 48\right]$. These data provide further evidence that DNA demethylation of myelin-related genes allows SCs to build myelin sheaths of appropriate thickness during development.

\section{Involvement of repressive histone methylation marks in} regulating myelination

Other repressive mechanisms mediated by $\mathrm{H} 3 \mathrm{~K} 27 \mathrm{me} 3$ marks on gene promoters have been reported during the myelination process. Ness and colleagues (2016) identify Nuc-ErbB3, a nuclear variant of ErbB3, as a DNA-binding protein that promotes $\mathrm{H} 3 \mathrm{~K} 27 \mathrm{me} 3$ at gene promoters presumably by activating the HMT EZH2. This study reports that loss of Nuc-ErbB3 (constitutive mouse mutant) leads to developmental hypermyelination [49]. Consistently, ablation of the PRC2 subunit Eed in immature SCs (using floxed Eed and PO-Cre mouse lines), that leads to inactivation of the PRC2 complex (mediating H3K27me3 through its HMT EZH2), results in hypermyelination in adults $\left[50^{\circ}\right]$. This is due to impaired repression of $I g f b p 2$ that maintains the activation of Akt-dependent myelination [50 ${ }^{\circ}$. However, no defect in developmental myelination occurs in Eed mutants $\left[50^{\circ}\right]$. A third study conducted in SC cultures proposes that loss of $\mathrm{EZH} 2$ leads in contrast to the inhibition of the myelination process through impaired inactivation of p75kip2 transcription that promotes expression of the inhibitor of myelination Hes5 [51]. These inconsistencies need to be resolved with additional in vivo studies.

\section{Functions of chromatin-remodeling enzymes in SCs after lesion}

Regenerative properties of the PNS after lesion are to a large extent due to the plasticity of SCs that first dedifferentiate and convert into repair cells in response to injury to foster and guide axonal regrowth, and second redifferentiate to remyelinate regenerated axons $[3,5,7,10]$. These different key steps of the regeneration process involve the dynamic regulation of many genes and thus the timed action of several transcription factors, which are known to promote either SC de-differentiation or redifferentiation after lesion. In comparison, our knowledge on the functions of chromatin-remodeling enzymes and their coordinated action with transcription factors after lesion is just emerging with recently published studies that are discussed below and illustrated in Figure 2.

HATs, HDACs, HMTs and HDMs control the conversion into repair cells, their cell cycle and functions after lesion

In adult nerves, injury-induced genes are silenced by the PRC2 complex, which adds repressive H3K27me3 marks 
at their promoter $\left[52^{\circ}\right]$. Upon injury, these genes are derepressed by $\mathrm{H} 3 \mathrm{~K} 27$ demethylation and activated by H3K4 methylation at promoter regions and by H3K27 acetylation at enhancers, the latter correlating with recruitment of the transcription factor cJun $\left[52^{\circ}, 53\right]$, a major inducer of SC conversion into repair cells [54]. This mechanism allows the expression of injury-induced genes including $S h h$ and the neurotrophic factor $G d n f\left[52^{\circ}\right]$, and thus promotes axonal regrowth after lesion.

De-differentiated SCs re-enter the cell cycle after injury. To prevent SC overproliferation that could lead to tumor formation, the Arf/Ink 4 locus is de-repressed by the HDM JMJD3 that demethylates H3K27 at the promoter of p19Arf and p16Ink4a and potentially also at the p15Ink4b promoter. These tumor-supressor proteins then inactivate SC proliferation [55].

Even though the conversion of SCs into repair cells after lesion is efficient, it is interesting to point out that this process is not optimal and can be improved. Indeed, shortly after injury, HDAC2 is upregulated in SCs and mediates the assembly of a protein complex with the two H3K9 HDMs KDM3A and JMJD2C and the transcription factor Sox10, which is recruited to the Oct6 SCE to demethylate H3K9 and induce Oct6 transcription. In turn, Oct6 slows down the upregulation of cJun [56 $\left.{ }^{\bullet \bullet}\right]$. Consistently, ablation of HDAC1/2 in adult SCs (using the tamoxifen-inducible P0-CreERT2 mouse line) or a short-term treatment with the HDAC1/2 inhibitor Mocetinostat delays Oct6 upregulation after lesion and leads to higher and earlier cJun upregulation, earlier conversion into repair cells and faster axonal regrowth $\left[56^{\bullet \bullet}\right]$.

\section{HDAC1/2 and the NuRD complex promote remyelination} after lesion

At the remyelination stage, the same protein complex assembled by HDAC2 after lesion (described above) is recruited to the Krox20 MSE to demethylate H3K9 and activate Krox 20 transcription [56 $6^{\circ}$ ]. Krox20 then induces remyelination. In the absence of HDAC1/2 in adult SCs, Krox20 expression is strongly reduced. This results in impaired remyelination $\left[56^{\circ}\right]$, indicating that HDAC1/2 are necessary for the remyelination process after lesion. Of note, a short-term HDAC1/2 inhibitor treatment after lesion (during the 3 days following lesion) does not interfere with the remyelination process, but leads to faster functional recovery due to faster and more efficient axonal regeneration, as mentioned above $\left[56^{\bullet \bullet}\right]$, thereby identifying a novel treatment to accelerate PNS regeneration after lesion.

To enable remyelination after lesion, it is also necessary to downregulate inhibitors of myelination such as Sox2, Hey2 and Id2. Similar to its function in developing SCs, the transcription factor Zeb2 represses the promoter of these genes in conjunction with the NuRD complex to silence their expression and thereby allow remyelination $\left[44^{\bullet \bullet}, 45^{\bullet \bullet}\right]$.

\section{Conclusion}

In summary, each step of SC development, the maintenance of integrity in adult nerves and the regeneration process after a PNS lesion are controlled by chromatinremodeling enzymes that either modify histones, DNA or remodel nucleosomes. The recent studies discussed in this review show that several chromatin-remodeling enzymes or events act in sync to regulate one cellular process. It appears also increasingly obvious that chromatin-remodeling enzymes are often targeted to a specific set of genes through their interaction with transcription factors, and thereby act as co-factors of these transcription factors to enable the regulation of their target genes.

Although recent findings have improved our understanding of chromatin-remodeling enzymes functions in SC development, maintenance and plasticity, a lot more work - preferentially carried out in vivo - is necessary to fully understand their functions and mechanisms of action, resolve inconsistencies, and potentially use and control them in disease contexts. In particular, it will be of major importance in future studies to analyze the coordinated action of different types of chromatin-remodeling enzymes, and elucidate their mode of activation and recruitment to specific target genes. Mass spectrometry combined with chromatin immunoprecipitation analyses will certainly reveal instrumental to identify dynamic protein complex formation and recruitment to specific loci depending on the developmental or regeneration stage.

\section{Conflict of interest statement}

Nothing declared.

\section{Acknowledgements}

Research in the Jacob lab is supported by the Swiss National Science Foundation (grant numbers: PP00P3_163759 and 31003A_173072), the International Foundation for Research in Paraplegia/OPO-Stiftung (grant number: IRP-P147), the Novartis Foundation (grant number: 15C191), and the Forschungspool of Fribourg University.

\section{References and recommended reading}

Papers of particular interest, published within the period of review, have been highlighted as:

- of special interest

$\bullet$ of outstanding interest

1. Graham A: The neural crest. Curr Biol 2003, 13:R381-R384.

2. Green SA, Simoes-Costa M, Bronner ME: Evolution of vertebrates as viewed from the crest. Nature 2015, 520:474482 .

3. Jessen KR, Mirsky R, Lloyd AC: Schwann cells: development and role in nerve repair. Cold Spring Harb Perspect Biol 2015, 7 a020487.

4. Jessen KR, Mirsky R: The origin and development of glial cells in peripheral nerves. Nat Rev Neurosci 2005, 6:671-682. 
5. Brosius Lutz A, Barres BA: Contrasting the glial response to axon injury in the central and peripheral nervous systems. Dev Cell 2014, 28:7-17.

6. Gomez-Sanchez JA, Carty L, Iruarrizaga-Lejarreta M, PalomoIrigoyen M, Varela-Rey M, Griffith M, Hantke J, Macias-Camara N, Azkargorta M, Aurrekoetxea I et al.: Schwann cell autophagy, myelinophagy, initiates myelin clearance from injured nerves. J Cell Biol 2015, 210:153-168.

7. Jessen KR, Mirsky R: The repair Schwann cell and its function in regenerating nerves. J Physiol 2016, 594:3521-3531.

8. Napoli I, Noon LA, Ribeiro S, Kerai AP, Parrinello S, Rosenberg LH, Collins MJ, Harrisingh MC, White IJ, Woodhoo A et al.: A central role for the ERK-signaling pathway in controlling Schwann cell plasticity and peripheral nerve regeneration in vivo. Neuron 2012, 73:729-742.

9. Cattin AL, Burden JJ, Van Emmenis L, Mackenzie FE, Hoving JJ, Garcia Calavia N, Guo Y, McLaughlin M, Rosenberg LH, Quereda $V$ et al.: Macrophage-induced blood vessels guide Schwann cell-mediated regeneration of peripheral nerves. Ce/l 2015, 162:1127-1139.

10. Cattin AL, Lloyd AC: The multicellular complexity of peripheral nerve regeneration. Curr Opin Neurobiol 2016, 39:38-46.

11. Becker PB, Workman JL: Nucleosome remodeling and epigenetics. Cold Spring Harb Perspect Biol 2013, 5:a017905.

12. Wilson BG, Roberts $C W$ : SWI/SNF nucleosome remodellers and cancer. Nat Rev Cancer 2011, 11:481-492.

13. Lai AY, Wade PA: Cancer biology and NuRD: a multifaceted chromatin remodelling complex. Nat Rev Cancer 2011, 11:588596

14. Smith ZD, Meissner A: DNA methylation: roles in mammalian development. Nat Rev Genet 2013, 14:204-220.

15. van der Wijst MG, Venkiteswaran M, Chen $H, X u$ GL, Plösch $T$, Rots MG: Local chromatin microenvironment determines DNMT activity: from DNA methyltransferase to DNA demethylase or DNA dehydroxymethylase. Epigenetics 2015 10:671-676.

16. Hodawadekar SC, Marmorstein R: Chemistry of acetyl transfer by histone modifying enzymes: structure, mechanism and implications for effector design. Oncogene 2007, 26:5528-5540.

17. Deckert J, Struhl K: Histone acetylation at promoters is differentially affected by specific activators and repressors. Mol Cell Biol 2001, 21:2726-2735.

18. Wang Z, Zang C, Cui K, Schones DE, Barski A, Peng W, Zhao K: Genome-wide mapping of HATs and HDACs reveals distinct functions in active and inactive genes. Cell 2009, 138:10191031.

19. Greer CB, Tanaka Y, Kim YJ, Xie P, Zhang MQ, Park IH, Kim TH: Histone deacetylases positively regulate transcription through the elongation machinery. Cell Rep 2015, 13:14441455.

20. Glozak MA, Sengupta N, Zhang X, Seto E: Acetylation and deacetylation of non-histone proteins. Gene 2005, 363:15-23.

21. Jacob C, Lebrun-Julien F, Suter U: How histone deacetylases control myelination. Mol Neurobiol 2011, 44:303-312.

22. Pattaroni $\mathrm{C}$, Jacob $\mathrm{C}$ : Histone methylation in the nervous system: functions and dysfunctions. Mol Neurobiol 2013, 47:740-756

23. Weider $M$, Wegner $M$ : SoxE factors: transcriptional regulators of neural differentiation and nervous system development. Semin Cell Dev Biol 2017, 63:35-42.

24. Jacob C, Lötscher P, Engler S, Baggiolini A, Varum Tavares S, Brügger V, John N, Büchmann-Møller S, Snider PL, Conway SJ et al.: HDAC1 and HDAC2 control the specification of neural crest cells into peripheral glia. J Neurosci 2014, 34:6112-6122.

25. Hagedorn L, Suter U, Sommer L: P0 and PMP22 mark a multipotent neural crest-derived cell type that displays community effects in response to TGF-beta family factors Development 1999, 126:3781-3794.

26. Auerbach R: Analysis of the developmental effects of a lethal mutation in the house mouse. J Exp Zool 1954, 127:305-329.

27. Franz T: Defective ensheathment of motoric nerves in the Splotch mutant mouse. Acta Anat 1990, 138:246-253.

28. Antonellis A, Huynh JL, Lee-Lin SQ, Vinton RM, Renaud G, Loftus SK, Elliot G, Wolfsberg TG, Green ED, McCallion AS, Pavan WJ: Identification of neural crest and glial enhancers at the mouse Sox10 locus through transgenesis in zebrafish. PLoS Genet 2008, 4:e1000174.

29. Wahlbuhl M, Reiprich S, Vogl MR, Bösl MR, Wegner M: Transcription factor Sox10 orchestrates activity of a neural crest-specific enhancer in the vicinity of its gene. Nucleic Acids Res 2012, 40:88-101.

30. Kurtz A, Zimmer A, Schnütgen F, Brüning G, Spener F, Müller T: The expression pattern of a novel gene encoding brain-fatty acid binding protein correlates with neuronal and glial cell development. Development 1994, 120:2637-2649.

31. Jacob C, Christen CN, Pereira JA, Somandin C, Baggiolini A, Lötscher P, Ozçelik M, Tricaud N, Meijer D, Yamaguchi T et al.: HDAC1 and HDAC2 control the transcriptional program of myelination and the survival of Schwann cells. Nat Neurosci 2011, 14:429-436.

32. Ghislain J, Desmarquet-Trin-Dinh C, Jaegle M, Meijer D, Charnay $\mathrm{P}$, Frain $\mathrm{M}$ : Characterisation of cis-acting sequences reveals a biphasic, axon-dependent regulation of Krox20 during Schwann cell development. Development 2002, 129:155166.

33. Topilko P, Schneider-Maunoury S, Levi G, Baron-Van Evercooren A, Chennoufi AB, Seitanidou T, Babinet C, Charnay P: Krox-20 controls myelination in the peripheral nervous system. Nature 1994, 371:796-799.

34. Brügger V, Engler S, Pereira JA, Ruff S, Horn M, Welzl H, Münger E, Vaquié A, Sidiropoulos PN, Egger B et al.: HDAC1/2-dependent PO expression maintains paranodal and nodal integrity independently of myelin stability through interactions with neurofascins. PLoS Biol 2015, 13:e1002258.

35. Chen $Y$, Wang $H$, Yoon SO, Xu X, Hottiger MO, Svaren J, Nave KA, Kim HA, Olson EN, Lu QR: HDAC-mediated deacetylation of NF$\kappa \mathrm{B}$ is critical for Schwann cell myelination. Nat Neurosci 2011, $14: 437-441$

36. Morton PD, Dellarole A, Theus MH, Walters WM, Berge SS, Bethea JR: Activation of NF-кB in Schwann cells is dispensable for myelination in vivo. J Neurosci 2013, 33:99329936.

37. Limpert AS, Bai S, Narayan M, Wu J, Yoon SO, Carter BD, Lu QR: NF-кB forms a complex with the chromatin remodeler BRG to regulate Schwann cell differentiation. J Neurosci 2013 33:2388-2397.

38. Weider M, Küspert M, Bischof M, Vogl MR, Hornig J, Loy K, Kosian T, Müller J, Hillgärtner S, Tamm ER et al.: Chromatinremodeling factor Brg1 is required for Schwann cell differentiation and myelination. Dev Cell 2012, 23:193-201.

39. Jagalur NB, Ghazvini M, Mandemakers W, Driegen S, Maas A, Jones EA, Jaegle M, Grosveld F, Svaren J, Meijer D: Functional dissection of the Oct6 Schwann cell enhancer reveals an essential role for dimeric Sox10 binding. J Neurosci 2011, 31:8585-8594.

40. Marathe HG, Mehta G, Zhang X, Datar I, Mehrotra A, Yeung KC, de la Serna IL: SWI/SNF enzymes promote SOX10-mediated activation of myelin gene expression. PLoS One 2013, 8 : e69037.

41. Mager GM, Ward RM, Srinivasan R, Jang SW, Wrabetz L, Svaren J: Active gene repression by the Egr2.NAB complex during peripheral nerve myelination. J Biol Chem 2008, 283:18187-18197.

42. Srinivasan R, Mager GM, Ward RM, Mayer J, Svaren J: NAB2 represses transcription by interacting with the $\mathrm{CHD} 4$ subunit 
of the nucleosome remodeling and deacetylase (NuRD) complex. J Biol Chem 2006, 281:15129-15137.

43. Hung $\mathrm{H}$, Kohnken $\mathrm{R}$, Svaren J: The nucleosome remodeling and deacetylase chromatin remodeling (NuRD) complex is required for peripheral nerve myelination. J Neurosci 2012 , 32:1517-1527.

44. Wu LM, Wang J, Conidi A, Zhao C, Wang H, Ford Z, Zhang L,

- Zweier C, Ayee BG, Maurel P et al.: Zeb2 recruits HDAC-NuRD to inhibit Notch and controls Schwann cell differentiation and remyelination. Nat Neurosci 2016, 19:1060-1072.

This study (together with [40]) identifies the transcription factor Zeb2 as a major repressor of inhibitors of myelination including Sox 2 and the Notch effector Hey2. Zeb2 function is essential in SCs, radial sorting, developmental myelination and remyelination after lesion being impaired in the absence of this transcription factor. The authors show that Zeb2 represses Sox2 and Hey2 by recruiting the NuRD complex.

45. Quintes S, Brinkmann BG, Ebert M, Fröb F, Kungl T, Arlt FA,

-• Tarabykin V, Huylebroeck D, Meijer D, Suter U et al.: Zeb2 is essential for Schwann cell differentiation, myelination and nerve repair. Nat Neurosci 2016, 19:1050-1059.

This study (together with [39]) shows that Zeb2 is essential for radia sorting, SC myelination and remyelination after injury. The authors show that Zeb2 represses the transcription of inhibitors of myelination including Sox2, Hey2 and Ednrb during development. During remyelination after lesion, the authors find persistence of Sox2, Hey2 and Id2 expression in Zeb2 mutant peripheral nerves.

- 46. Varela-Rey M, Iruarrizaga-Lejarreta M, Lozano JJ, Aransay AM,

-• Fernandez AF, Lavin JL, Mósen-Ansorena D, Berdasco M, Turmaine M, Luka Z et al.: S-adenosylmethionine levels regulate the Schwann cell DNA methylome. Neuron 2014, 81:1024-1039.

In this paper, the authors show that SCs undergo global DNA demethylation during the myelination process. This correlates with the downregulation of DNMTs and of the methyl donor SAMe and the upregulation of proteins promoting DNA demethylation. In mutant mice for GNMT that transforms SAMe into S-adenosylhomocysteine (SAH) and N-methylglycine, the availability of SAMe and DNA methylation are increased, and myelin is thinner, showing that DNA demethylation is necessary for appropriate myelination.

47. Niehrs C, Schäfer A: Active DNA demethylation by Gadd45 and DNA repair. Trends Cell Biol 2012, 22:220-227.

48. Luka Z, Capdevila A, Mato JM, Wagner C: A glycine Nmethyltransferase knockout mouse model for humans with deficiency of this enzyme. Transgenic Res 2006, 15:393-397.

49. Ness JK, Skiles AA, Yap EH, Fajardo EJ, Fiser A, Tapinos N: NucErbB3 regulates H3K27me3 levels and HMT activity to establish epigenetic repression during peripheral myelination. Glia 2016, 64:977-992.

50. Ma KH, Hung HA, Srinivasan R, Xie H, Orkin SH, Svaren J:

- Regulation of peripheral nerve myelin maintenance by gene repression through Polycomb Repressive Complex 2. J Neurosci 2015, 35:8640-8652.

This study identifies the PRC2 complex as a necessary myelination break in adult nerves. Indeed, in vivo inactivation of the PRC2 complex leads to hypermyelination in adult nerves, whereas developmental myelination is not affected. The authors show that the PRC2 complex silences the Igfbp2 promoter by H3K27me3, which prevents Igfbp2-induced activation of Akt and subsequent overmyelination.

51. Heinen A, Tzekova N, Graffmann N, Torres KJ, Uhrberg M, Hartung HP, Küry P: Histone methyltransferase enhancer of zeste homolog 2 regulates Schwann cell differentiation. Glia 2012, 60:1696-1708.

52. Ma KH, Hung HA, Svaren J: Epigenomic regulation of Schwann - cell reprogramming in peripheral nerve injury. J Neurosci 2016, 36:9135-9147.

This article highlights the switch in histone methylation marks occurring at the promoters and enhancers of injury-induced genes after lesion. The authors show that the promoter of these genes is repressed by H3K27me3 in adult nerves and is de-repressed by H3K27 demethylation and activated by $\mathrm{H} 3 \mathrm{~K} 4 \mathrm{me} 3$ upon lesion. Simultaneously, enhancers of these genes are activated by H3K27 acetylation, which correlates with the recruitment of cJun. These genes include Shh and the neurotrophic factor Gdnf.

53. Hung HA, Sun G, Keles S, Svaren J: Dynamic regulation of Schwann cell enhancers after peripheral nerve injury. J Biol Chem 2015, 290:6937-6950.

54. Arthur-Farraj PJ, Latouche M, Wilton DK, Quintes S, Chabrol E, Banerjee A, Woodhoo A, Jenkins B, Rahman M, Turmaine M et al. c-Jun reprograms Schwann cells of injured nerves to generate a repair cell essential for regeneration. Neuron 2012, 75:633647.

55. Gomez-Sanchez JA, Gomis-Coloma C, Morenilla-Palao C Peiro G, Serra E, Serrano M, Cabedo H: Epigenetic induction of the Ink4a/Arf locus prevents Schwann cell overproliferation during nerve regeneration and after tumorigenic challenge. Brain 2013, 136:2262-2278.

56. Brügger V, Duman M, Bochud M, Münger E, Heller M, Ruff S,

-• Jacob C: Delaying histone deacetylase response to injury accelerates conversion into repair Schwann cells and nerve regeneration. Nat Commun 2017, 8:14272.

This study shows that HDAC2 assembles a protein complex with the two H3K9 HDMs KDM3A and JMJD2C and with Sox10 to de-repress and activate Oct6 transcription early after a PNS lesion. This complex is subsequently recruited to the Krox20 MSE to de-repress and activate Krox20 transcription at the remyelination stage. The authors demonstrate that inactivating this mechanism accelerates cJun upregulation, the conversion into repair cells and axonal regrowth, but impairs remyelination. However, short-term inactivation using an HDAC1/2 inhibitor accelerates regeneration and functional recovery without impairing remyelination, thereby identifying a novel treatment to improve PNS regeneration after lesion. 